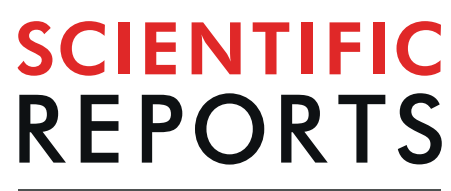

natureresearch

\title{
OPEN The Two-Species Model of transketolase explains donor substrate-binding, inhibition and heat-activation
}

\begin{abstract}
Henry C. Wilkinson (1) \& Paul A. Dalby (i)*
We recently characterised a low-activity form of $E$. coli transketolase, $\mathrm{TK}_{\text {low, }}$ which also binds the cofactor thiamine pyrophosphate (TPP) with an affinity up to two-orders of magnitude lower than the previously known high TPP-affinity and high-activity form, $\mathrm{TK}_{\text {high }}$ in the presence of $\mathrm{Mg}^{2+}$. We observed previously that partial oxidation was responsible for increased $\mathrm{TK}_{\text {high }}$ activity, while lowactivity $\mathrm{TK}_{\text {low }}$ was unmodified. In the present study, the fluorescence-based cofactor-binding assay was adapted to detect binding of the $\beta$-hydroxypyruvate (HPA) donor substrate to wild-type transketolase and a variant, S385Y/D469T/R520O, that is active towards aromatic aldehydes. Transketolase HPA affinity again revealed the two distinct forms of transketolase at a $\mathrm{TK}_{\text {high }}: \mathrm{TK}_{\text {low }}$ ratio that matched those observed previously via TPP binding to each variant. The HPA dissociation constant of $\mathrm{TK}_{\text {low }}$ was comparable to the substrate-inhibition dissociation constant, $K_{i}^{H P A}$, determined previously. We provide evidence that $K_{i}^{H P A}$ is a convolution of binding to the low-activity $\mathrm{TK}_{\text {low }}-\mathrm{TK}_{\text {low }}$ dimer, and the $\mathrm{TK}_{\text {low }}$ subunit of the partially-active $\mathrm{TK}_{\text {high }}-\mathrm{TK}_{\text {low }}$ mixed dimer, where HPA binding to the $\mathrm{TK}_{\text {low }}$ subunit of the mixed dimer results in inhibition of the active $\mathrm{TK}_{\mathrm{h}}$ sh subunit. Heat-activation of transketolase was similarly investigated and found to convert the $\mathrm{TK}_{\text {low }}$ subunit of the mixed dimer to have $\mathrm{TK}_{\text {high }}$-like properties, but without oxidation.
\end{abstract}

Transketolase is a key enzyme of the pentose phosphate pathway (PPP), is ubiquitous in all organisms, and provides a unique link between glycolysis and the non-oxidative phase of the PPP. Transketolase is a thiamine pyrophosphate (TPP)-dependent enzyme that reversibly transfers a two-carbon ketol group from a donor substrate (usually a five-carbon ketose) to an acceptor aldehyde substrate (usually a five-carbon aldose) via a ping-pong reaction mechanism, forming a new asymmetric $\mathrm{C}-\mathrm{C}$ bond with high regio- and stereo-specificity. In biocatalysis, $\beta$-hydroxypyruvate (HPA) is often used as the donor substrate due to the irreversible, concomitant release of $\mathrm{CO}_{2}$ as a by-product. Strong substrate inhibition has been observed above $25 \mathrm{mM}$ HPA with an inhibition constant of around $42 \mathrm{mM}^{1,2}$. The cause of this substrate inhibition is currently unknown and is addressed in this study.

The inactive, apo-form of transketolase is in a monomer-dimer equilibrium that is dependent on protein concentration. Upon cofactor binding, both the inactive apo-monomer and apo-dimer are converted into the catalytically active, dimeric holo-form of, until recently, seemingly structurally-identical subunits, with two active-sites per homodimer, located at the subunit interface ${ }^{3,4}$. Each active site is comprised of one divalent cation, such as $\mathrm{Mg}^{2+}$, and one TPP molecule. In the S. cerevisiae transketolase apo-dimer, even after removal of free $\mathrm{Ca}^{2+}$, one $\mathrm{Ca}^{2+}$ ion was bound extremely tightly to one active site and could only be removed using harsh treatment, while the second $\mathrm{Ca}^{2+}$ ion dissociated easily. Subsequent addition of $\mathrm{Mg}^{2+}$ to the medium led to a transketolase dimer with one $\mathrm{Ca}^{2+}$ - and one $\mathrm{Mg}^{2+}$-reconstituted active site, with positive cooperativity observed between active sites upon TPP-binding ${ }^{5}$. It is unclear if E. coli transketolase behaves in a similar way, since both positive and negative cooperativity have been observed at different $\left[\mathrm{Mg}^{2+}\right]^{6}$.

Transketolase displays ping-pong kinetics and catalyses two sequential half-reactions; formation of the dihydroxyethyl-TPP (DHE-TPP) carbanion intermediate, followed by transfer of this two-carbon ketol group from the carbanion intermediate to an acceptor aldehyde, thus returning the enzyme to its starting state. It is 
thought that there is considerable communication between active sites in order to coordinate the ping-pong kinetics between active sites, such that catalysis alternates between active sites, giving rise to the 'half-of-the-sites reactivity' phenomenon that has been observed in transketolase ${ }^{7}$ and other TPP-dependent enzymes ${ }^{8-11}$.

There is evidence that in the E1 subunit of the pyruvate dehydrogenase complex (PDC) (EC 1.2.4.1), the two TPP-containing active-sites communicate via a $20 \AA$ proton wire that shuttles a proton between active sites, enabling the cofactors to operate reciprocally as general acid-base catalytic moieties, thus synchronising the ping-pong mechanism across active sites ${ }^{12}$. It was suggested by the authors that many thiamine-dependent enzymes may function in a similar way. Recently, the expected structural asymmetry between the two monomers of apo-dimeric E. coli TK was finally observed at the proton scale and was implicated as a key feature of the proton wire and hence cooperativity between active sites $^{13}$.

Transketolase is unusual in that incubation at between $40-55^{\circ} \mathrm{C}$ for $1 \mathrm{~h}$ increases the residual activity significantly, when measured after re-cooling the samples to $25^{\circ} \mathrm{C}$. For example, incubation at $42^{\circ} \mathrm{C}$ for $1 \mathrm{hr}$ increases activity by $50 \%{ }^{14,15}$. It was postulated that this curious phenomenon may be the result of an inactive form of transketolase being physically altered or activated by temperature, although no such species had been detected at that time.

Until recently, it was thought that purified transketolase existed in a single form with a high affinity for TPP, which is essential for catalytic activity. However, we previously found that transketolase exists with two distinct subpopulations of subunits in purified samples, $\mathrm{TK}_{\mathrm{high}}$ and $\mathrm{TK}_{\text {low }}$ with over 200 -fold different affinities for $\mathrm{TPP}$ at high $\left[\mathrm{Mg}^{2+}\right]^{6}$. TK $\mathrm{high}$ was found to form via at least one specific oxidation of the unmodified $\mathrm{TK}_{\mathrm{low}}$. The $\mathrm{TK}_{\mathrm{low}}$ subunit had only $4.5 \%$ of the activity of $\mathrm{TK}_{\text {high }}$ in the presence of saturating concentrations of TPP, and was also found to have disrupted the cooperativity between TPP-binding sites observed for $\mathrm{TK}_{\text {high }}$. The two distinct monomeric subunits were found to combine into at least two distinct dimer forms, the $\mathrm{TK}_{\mathrm{high}}-\mathrm{TK}_{\mathrm{high}}$ and $\mathrm{TK}_{\mathrm{low}}-\mathrm{TK}_{\mathrm{low}}$ dimers, while the $\mathrm{TK}_{\mathrm{high}}-\mathrm{TK}_{\text {low }}$ mixed dimer was not observed directly, but not ruled out as possible.

The fraction of TPP that can bind to $\mathrm{TK}_{\text {high }}$ relative to $\mathrm{TK}_{\text {low }} \% B_{\max (h i g h)}{ }^{T P P}(33.6 \pm 2.9 \%)$, was found to be invariant to changes in $\left[\mathrm{Mg}^{2+}\right]$ and $[\mathrm{TK}]$, while the addition of thiamine during fermentation to increase cellular [TPP] also had no effect. It was hypothesized that oxidative stress may play a role in determining the ratio of $\mathrm{TK}_{\mathrm{high}}: \mathrm{TK}_{\mathrm{low}}$, and detection of post-translational oxidations by mass spectrometry in $\mathrm{TK}_{\mathrm{high}}$ supported our hypothesis ${ }^{6}$.

The pentose phosphate pathway runs parallel to glycolysis and has several cellular functions, including the generation of pentose sugars as well as ribose-5-phoshate, the latter the precursor for nucleotide biosynthesis. It is therefore a key branch-point in the diversion of metabolic flux towards biosynthetic pathways. However, one of the PPP's most important utilities is to respond to and negate oxidative stress, often due to reactive oxygen species (ROS), through production of an anti-oxidant, $\mathrm{NADPH}^{16}$.

Intracellular NADPH is continuously used as a reducing agent to replenish the reduced glutathione pool to protect against oxidative stress and to maintain a stable cellular redox potential ${ }^{17-19}$. Recently, it has been demonstrated that in the short-term, oxidative stress can be mediated by redox-sensitive enzymes in lower glycolysis $^{20-23}$. In these instances, oxidative post-translational modifications (PTMs) can provide regulation with a rapid response time by cysteine oxidation and subsequent diversion of glycolytic flux through the PPP to generate NADPH and nucleotide precursors for DNA repair. Another separate cysteine-based regulatory system that protects against oxidative stress by diverting flux from $\mathrm{NAD}^{+}$-dependent ALDS to NADH-producing ALDHs was also recently elucidated ${ }^{24}$. While a few control points have been identified in lower glycolysis ${ }^{20-23}$ and in the oxidative phase of the $\mathrm{PPP}^{16}$, to the authors' knowledge redox regulation of enzymes in the non-oxidative phase of the PPP are yet to be identified or fully characterised. The identification of such regulatory mechanisms may also inform studies into the role of transketolase in tumour progression, given its increased activity found in many cancer cells ${ }^{25}$.

Here, we examine directly the binding of substrate to the transketolase species subpopulations for both wild type and the variant S385Y/D469T/R520Q, and provide further evidence that transketolase is a redox-regulated enzyme, that could therefore potentially play a major role in the control of flux through the PPP during oxidative stress. The new substrate inhibition and heat-activation data indicated that a mixed dimer species, $\mathrm{TK}_{\mathrm{high}}-\mathrm{TK}_{\mathrm{low}}$, may be the cause of donor-substrate inhibition and heat-activation. Finally, we propose an updated Two-Species Model for transketolase activation, regulation and inhibition that includes explanations of the origin of $\% B_{\max (h i g h)}$, HPA inhibition, active-site cooperativity, redox control and heat-shock activation.

\section{Results}

The Two-species Transketolase model is persistent across variants. Previously, we measured the TPP-binding parameters of wild-type transketolase over a range of cofactor concentrations ${ }^{6}$. Here, we report the TPP-binding parameters for S385Y/D469T/R520Q, a variant that was previously engineered for activity towards aromatic aldehydes ${ }^{26}$. The rationale behind these measurements was to (a) confirm the existence of both $\mathrm{TK}_{\text {high }}$ and $\mathrm{TK}_{\mathrm{low}}$ across variants; and (b) to demonstrate a direct correlation between changes in the \% substrate inhibi-

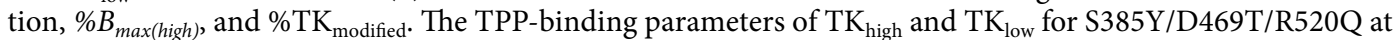
between $0-18 \mathrm{mM} \mathrm{Mg}^{2+}$ are summarised below (Fig. 1a-d; Tables 1 and 2; Fig. S1, Supplementary Information). The double-Hill function ${ }^{6}$ was again utilised to determine the independent TPP-binding parameters for $\mathrm{TK}_{\text {high }}$ and $\mathrm{TK}_{\mathrm{low}}$. As previously for wild-type $\mathrm{TK}^{6}$, at $0 \mathrm{mM} \mathrm{Mg}^{2+}$, the binding parameters of $\mathrm{TK}_{\mathrm{high}}$ and $\mathrm{TK}_{\mathrm{low}}$ for $\mathrm{S} 385 \mathrm{Y} /$ D469T/R520Q were too similar to deconvolve with accuracy (Fig. S2, Supplementary Information), therefore the dissociation constants and Hill coefficients were constrained to equal each other within the double-Hill function.

The trends in the TPP-binding parameters of $\mathrm{TK}_{\text {high }}$ and $\mathrm{TK}_{\mathrm{low}}$ of S385Y/D469T/R520Q were similar to those of wild-type transketolase ${ }^{6}$. A significant decrease and comparatively small increase in the dissociation constants were observed for $\mathrm{TK}_{\text {high }}$ (Fig. 1a) and $\mathrm{TK}_{\text {low }}$ (Fig. 1b), respectively, at higher $\left[\mathrm{Mg}^{2+}\right]$. However, $\mathrm{TK}_{\text {high }}$ and $\mathrm{TK}_{\text {low }}$ of S385Y/D469T/R520Q each bound to TPP with a lower affinity than the wild-type (4.5-fold and 1.6-fold 

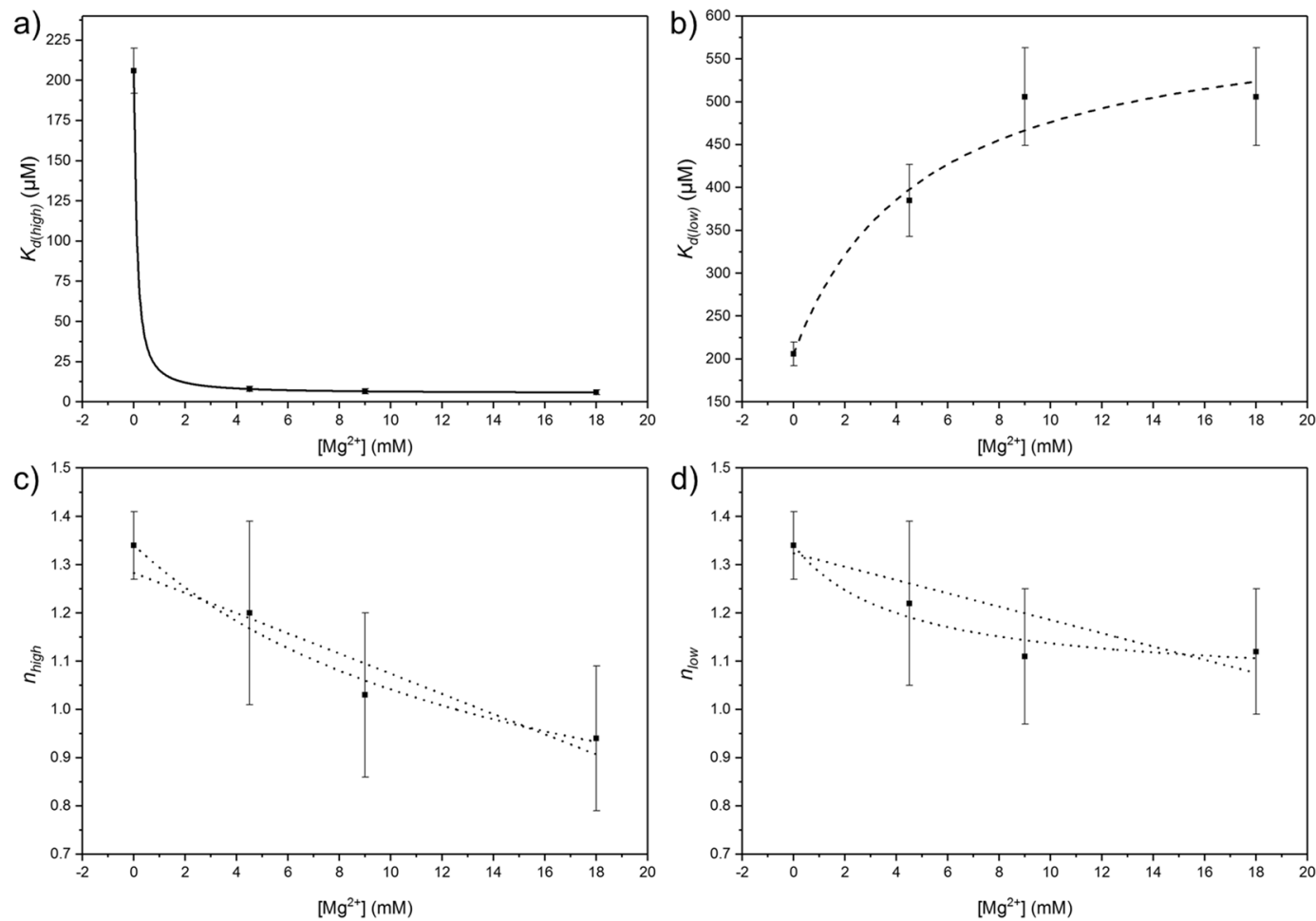

Figure 1. Dependence of TPP-dissociation constants and Hill coefficients of the high and low affinity binding sites for $0.05 \mathrm{mg} / \mathrm{mL}$ TK (S385Y/D469T/R520Q) at $0 \mathrm{mM}, 4.5 \mathrm{mM}, 9 \mathrm{mM}$ and $18 \mathrm{mM} \mathrm{Mg}^{2+}$. (a,b) - the dissociation constants of the high and low affinity binding sites, respectively. (c,d) - the Hill coefficients of the high and low affinity binding sites, respectively. Solid lines represent quantified, fitted graphs; dashed lines represent clear, unquantified trends; dotted lines represent possible trends that fit the data. Associated errors are the fitting error when fitting to the double-Hill equation.

\begin{tabular}{|l|l|l|l|l|}
\hline $\begin{array}{l}{\left[\mathrm{Mg}^{2+}\right]} \\
(\mathrm{mM})\end{array}$ & $\begin{array}{l}\boldsymbol{K}_{d(\text { high })} \text { TPP } \\
(\boldsymbol{\mu M})\end{array}$ & \pm & $\boldsymbol{n}_{\text {high }}{ }^{\text {TPP }}$ & \pm \\
\hline 0 & 206 & 14 & 1.34 & 0.07 \\
\hline 4.5 & 8.03 & 1.59 & 1.20 & 0.19 \\
\hline 9 & 6.60 & 1.64 & 1.03 & 0.17 \\
\hline 18 & 5.96 & 1.52 & 0.94 & 0.15 \\
\hline
\end{tabular}

Table 1. Summary of the TPP-binding parameters of the high affinity binding sites of the $\mathrm{TK}_{\mathrm{high}}$ species of S385Y/D469T/R520Q. A TK concentration of $0.05 \mathrm{mg} / \mathrm{mL}$ was used in each binding assay. Associated errors are the fitting error when fitting to the double-Hill function.

\begin{tabular}{|l|l|l|l|l|}
\hline $\begin{array}{l}{\left[\mathrm{Mg}^{2+}\right]} \\
(\mathbf{m M})\end{array}$ & $\begin{array}{l}\boldsymbol{K}_{d(\text { low })}{ }^{T P P} \\
(\boldsymbol{\mu M})\end{array}$ & \pm & $\boldsymbol{n}_{\text {low }}{ }^{\text {TPP }}$ & \pm \\
\hline 0 & 206 & 14 & 1.34 & 0.07 \\
\hline 4.5 & 385 & 42 & 1.22 & 0.17 \\
\hline 9 & 506 & 57 & 1.11 & 0.14 \\
\hline 18 & 506 & 57 & 1.12 & 0.13 \\
\hline
\end{tabular}

Table 2. Summary of the TPP-binding parameters of the low affinity binding sites of the $\mathrm{TK}_{\mathrm{low}}$ species of S385Y/D469T/R520Q. A TK concentration of $0.05 \mathrm{mg} / \mathrm{mL}$ was used in each binding assay. Associated errors are the fitting error when fitting to the double-Hill function.

lower at $18 \mathrm{mM} \mathrm{Mg}^{2+}$, respectively), and TPP-binding to $\mathrm{TK}_{\text {high }}$ (Fig. 1c) was less cooperative in S385Y/D469T/ R520Q. Furthermore, the binding cooperativity changed from being positive, to non-cooperative as $\left[\mathrm{Mg}^{2+}\right]$ was increased, but no longer peaked at the physiologically relevant $\left[\mathrm{Mg}^{2+}\right]$ of $4 \mathrm{mM}$ as observed in wild-type TK . The trends were most likely non-linear in nature, but a linear relationship could not be ruled out (Fig. 1c,d). These results indicated that while changes in maximum affinity may change between variants, the response of $\mathrm{TK}_{\mathrm{high}}$ and $\mathrm{TK}_{\mathrm{low}}$ to $\mathrm{Mg}^{2+}$ was persistent across variants, although the cooperativity was impacted quite considerably. 

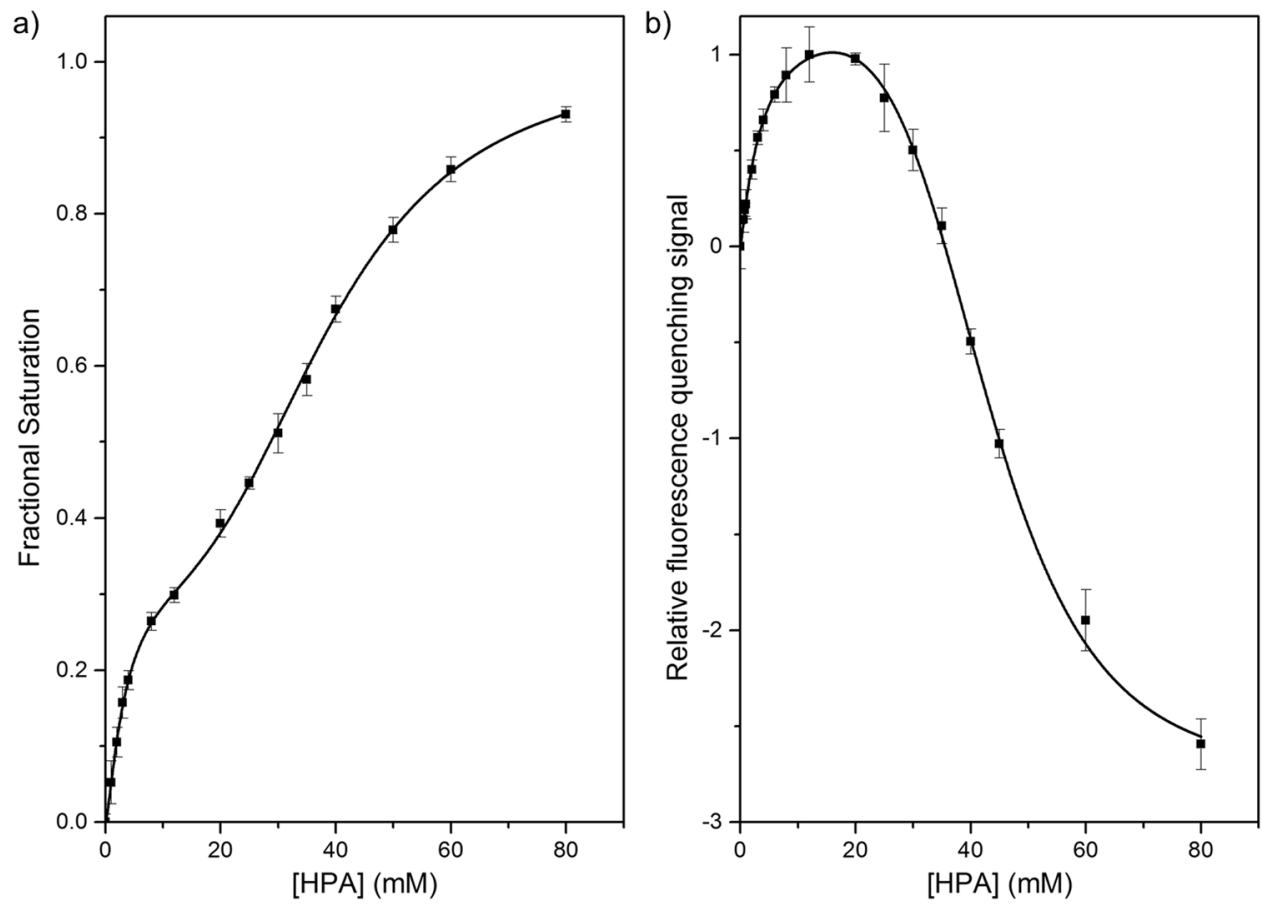

Figure 2. Experimental data of $0.05 \mathrm{mg} / \mathrm{mL}$ (a) wild-type and (b) S385Y/D469T/R520Q transketolase binding to HPA in the presence of $9 \mathrm{mM} \mathrm{Mg}^{2+}$ and $0.3 \mathrm{mM}$ TPP in $50 \mathrm{mM}$ Tris-HCl buffer. Experimental data was fitted to the double-Hill function ${ }^{6}$. The change in binding was reported as the 'relative fluorescence quenching signal' in (b) because of the change in signal from fluorescence quenching to increased fluorescence at above $20 \mathrm{mM}$ HPA. As such, normalisation to give fractional saturation is not possible in (b). Error bars correspond to the standard error of the mean, $n=5$.

\begin{tabular}{|l|l|l|l|l|l|l|l|l|}
\hline Variant & $\begin{array}{l}\boldsymbol{K}_{\text {d(high }} \text { HPA } \\
(\mathbf{m M})\end{array}$ & \pm & $\begin{array}{l}\boldsymbol{K}_{\text {d(low) }} \text { HPA } \\
(\mathbf{m M})\end{array}$ & \pm & $n_{\text {high }}{ }^{\text {HPA }}$ & \pm & $n_{\text {low }}{ }^{\text {HPA }}$ & \pm \\
\hline Wild-type & 3.42 & 0.45 & 39.3 & 0.7 & 1.39 & 0.19 & 3.14 & 0.18 \\
\hline S385Y/D469T/R520Q & 3.61 & 0.31 & 43.1 & 0.4 & 1.15 & 0.06 & 4.60 & 0.21 \\
\hline
\end{tabular}

Table 3. Summary of the binding parameters of the high and low affinity binding sites of $\mathrm{TK}_{\text {high }}$ and $\mathrm{TK}_{\mathrm{low}}$ of wild-type and S385Y/D469T/R520Q transketolase. Each binding assay used $0.05 \mathrm{mg} / \mathrm{mL}$ transketolase, $9 \mathrm{mM}$ $\mathrm{MgCl}_{2}$ and $0.3 \mathrm{mM} \mathrm{TPP}$ in $50 \mathrm{mM}$ Tris- $\mathrm{HCl}$ buffer. Associated errors are the fitting error when fitting to the double-Hill function.

Adaption of the fluorescence quenching-based TPP-binding assay for donor substrate binding. Similar to our previous fluorescence quenching-based cofactor-binding assay ${ }^{6}$, binding of HPA to holo-transketolase was shown to further quench the intrinsic fluorescence of holo-transketolase $\left(\lambda_{\mathrm{ex}}=240 \mathrm{~nm}\right.$; $\lambda_{\mathrm{em}}=330 \mathrm{~nm}$ ) (Fig. S3, Supplementary Information). Like TPP, HPA absorbed relatively strongly at $240 \mathrm{~nm}$. The sample signal was therefore also corrected for the inner filter effect (IFE) by generating a correction factor, determined empirically from the change in fluorescence intensity of $0-80 \mathrm{mM}$ free HPA in $50 \mathrm{mM}$ Tris- $\mathrm{HCl}$ buffer, $9 \mathrm{mM} \mathrm{Mg}^{2+}$ and $0.3 \mathrm{mM}$ TPP (Fig. S4, Supplementary Information).

Characterisation of $E$. coli transketolase binding to HPA. The dissociation constant of HPA-binding to transketolase, $K_{d}^{H P A}$, has never been previously determined directly, although the closely-related Michaelis-Menten constant of HPA-binding to wild-type transketolase, $K_{m}{ }^{H P A}$, has been reported as $5.5 \pm 0.5$ $\mathrm{mM}^{27}$ and $5.3 \mathrm{mM}^{28}$. Substrate inhibition by HPA, $K_{i}^{H P A}$, has also been measured as $42.2 \mathrm{mM}$ and $43 \mathrm{mM}^{1,2}$, providing suitable benchmarks for comparison. The double-Hill function ${ }^{6}$ was again used to determine the HPA-binding parameters for both wild-type transketolase and S385Y/D469T/R520Q, as the fit to the data was superior to a single Hill function (Fig. 2a-b; Table 3), as reported previously ${ }^{6}$.

Two distinct HPA-binding events, $\mathrm{TK}_{\text {high }}$ and $\mathrm{TK}_{\text {low }}$ were detected, with an 11 -fold difference in affinity, supporting our previously postulated Two-Species Model of transketolase. The three mutations within the S385Y/D469T/R520Q variant appeared to have no significant effect on the affinity of HPA binding, but significantly decreased the cooperativity of both TPP- and HPA-binding in $\mathrm{TK}_{\text {high }}$, and increased the cooperativity of HPA-binding in $\mathrm{TK}_{\text {low }}$ (Table 3). In addition, binding of HPA to $\mathrm{TK}_{\text {low }}$ resulted in a decrease in fluorescence quenching in S385Y/D469T/R520Q. This was presumably related to the introduction of the fluorescent tyrosine 


\begin{tabular}{|l|l|l|l|l|l|l|}
\hline Variant & $\% \boldsymbol{B}_{\max (\text { high }}{ }^{\text {TPP }}$ & \pm & $\% \boldsymbol{B}_{\max (\text { high }}{ }^{{ }^{P A}}$ & \pm & TK $_{\text {modified }}$ & \pm \\
\hline Wild-type & $33.6 \%$ & $2.9 \%$ & $33.7 \%$ & $3.0 \%$ & $31.0 \%$ & $1.7 \%$ \\
\hline S385Y/D469T/R520Q & $27.1 \%$ & $5.3 \%$ & $23.8 \%$ & $5.0 \%$ & $30.0 \%$ & $0.1 \%$ \\
\hline
\end{tabular}

Table 4. The $\% B_{\max (\text { high })}{ }^{T P P}$ and $\% B_{\max (\text { high })}{ }^{H P A}$ and $\% \mathrm{TK}_{\text {modified }}$ of wild-type transketolase ${ }^{6}$ and variant S385Y/ D469T/R520Q, determined by TPP-binding, HPA-binding and mass spectrometry data. Associated errors are either the the fitting error $\left(\% B_{\max (h i g h)}{ }^{T P P}\right.$ and $\left.\% B_{\max (h i g h)}{ }^{H P A}\right)$ or the standard error of the mean $(\% \mathrm{TK}$ modified, $\mathrm{n}=3$ ).

\begin{tabular}{|l|l|l|l|l|l|l|}
\hline Sample & $\begin{array}{l}\boldsymbol{K}_{\text {d(high) }} \\
(\boldsymbol{\mu M})\end{array}$ & \pm & $\boldsymbol{K}_{\text {d(low) }}(\boldsymbol{\mu M})$ & \pm & $\% B_{\max (\text { (high })}$ & \pm \\
\hline Wild-type & 2.28 & 0.23 & 276 & 23 & $33.6 \%$ & $2.9 \%$ \\
\hline Heat-activated wild-type & 1.75 & 0.26 & 157 & 28 & $51.7 \%$ & $5.1 \%$ \\
\hline
\end{tabular}

Table 5. Summary of the TPP-dissociation constants of wild-type $\mathrm{TK}_{\text {low }}$ and $\mathrm{TK}_{\text {high }}$ pre- and post-incubation at $42^{\circ} \mathrm{C}$ for $1 \mathrm{~h}$. A TK concentration of $0.05 \mathrm{mg} / \mathrm{mL}$ was used in each binding assay. Associated errors are the fitting error when fitting to the double-Hill equation.
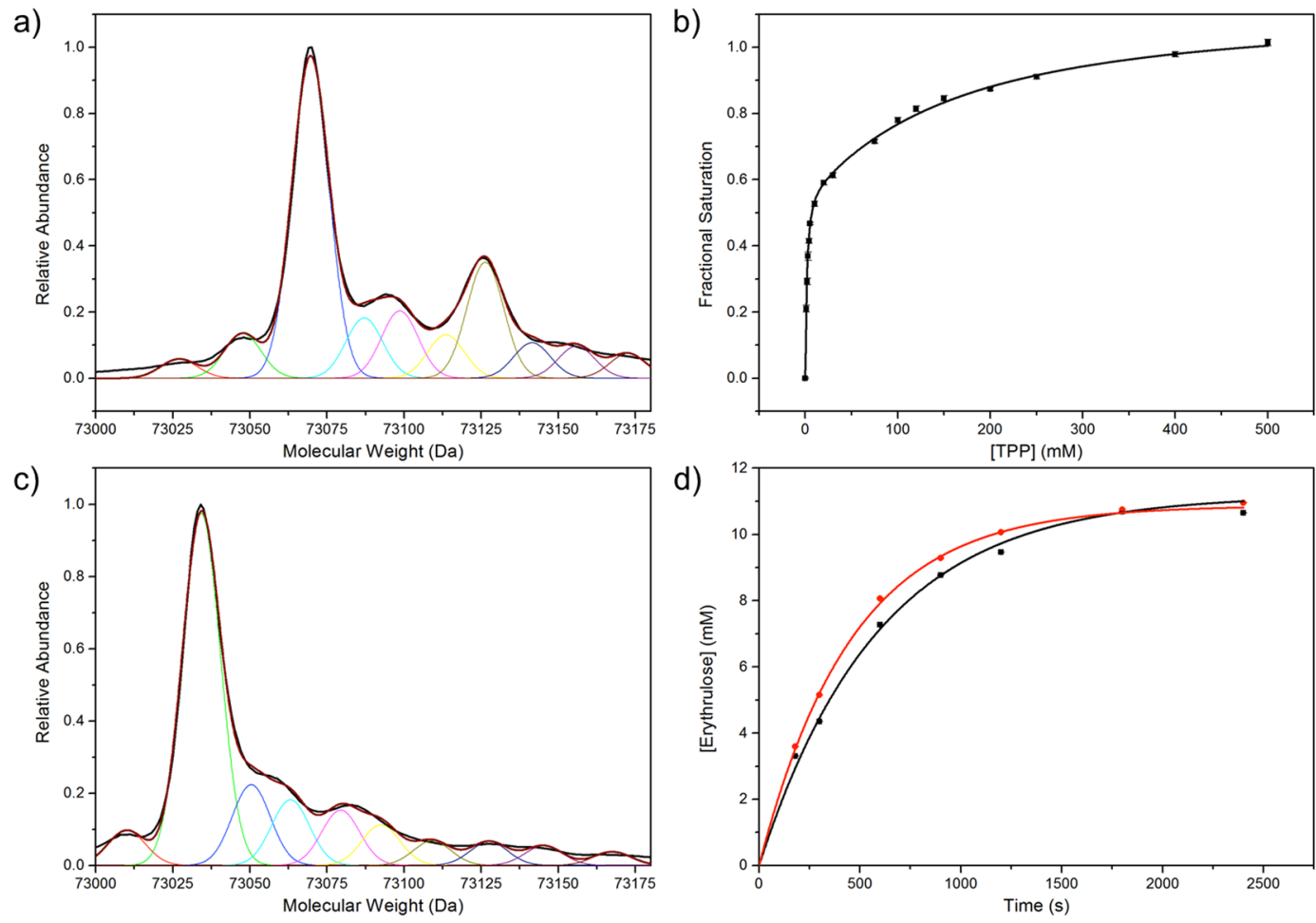

Figure 3. The impact of heat-activation on $\% \mathrm{TK}_{\text {high, }}$ TPP-binding and activity. The mass spectra of purified (a) S385/D469T/R520Q and (c) heat-activated wild-type TK $\left(1 \mathrm{hr}\right.$ at $\left.42^{\circ} \mathrm{C}\right)$. The major peak corresponded to unmodified TK, while the next two peaks corresponded to modified TK ${ }^{6}$. (b) TPP-binding to heat-activated $0.05 \mathrm{mg} / \mathrm{mL}$ wild-type $\mathrm{TK}\left(1 \mathrm{hr}\right.$ at $\left.42^{\circ} \mathrm{C}\right), 9 \mathrm{mM} \mathrm{Mg}^{2+}$. Error bars correspond to the standard error of the mean, $\mathrm{n}=5$. (d) Activity data of purified $0.067 \mathrm{mg} / \mathrm{mL}$ wild-type TK with $50 \mathrm{mM}$ GA and $50 \mathrm{mM} \mathrm{HPA}$, pre-incubated with $9 \mathrm{mM} \mathrm{Mg}^{2+}$ and $50 \mu \mathrm{M}$ TPP before (black) and after (red) heat-activation ( $1 \mathrm{hr}$ at $42^{\circ} \mathrm{C}$ ). Error bars correspond to the standard error of the mean, $\mathrm{n}=3$.

at residue 385. Importantly, the proportion of HPA bound to $\mathrm{TK}_{\text {high }}$ relative to all TK at saturation, $\% B_{\max (h i g h)}{ }^{H P A}$, matched that for TPP bound to $\mathrm{TK}_{\text {high }}, \% B_{\max (\text { high })}{ }^{T P P}$, for each variant (Tables 4 and 5), indicating that the structural difference between $\mathrm{TK}_{\mathrm{high}}$ and $\mathrm{TK}_{\text {low }}$ impacted both TPP and HPA binding, and confirmed that the two species remained distinct from each other. Furthermore, the $\% B_{\max (\text { high })}{ }^{H P A}$ and $\% B_{\max (h i g h)}{ }^{T P P}$ also matched the $\% \mathrm{TK}_{\text {modified }}$ determined from the mass spectra of wild-type transketolase ${ }^{6}$ and the variant S385Y/D469T/R520Q (Fig. 3a; Table 4), demonstrating that the enzyme affinity parameters and the oxidation to form $\mathrm{TK}_{\text {high }}$ were correlated.

The $K_{d}^{H P A}$ of wild-type $\mathrm{TK}_{\text {high }}, K_{d(\text { high })}{ }^{H P A}$, was slightly lower than the previously reported $K_{m}{ }^{H P A} 27,28$. This was expected because $K_{m}$ is measured via enzyme kinetics, and is therefore a chemical pseudo-equilibrium which is convoluted with the additional forward reaction for formation of product, in other words substrate turnover 
$\left(k_{\text {cat }}\right)$. By comparison, $K_{d}{ }^{H P A}$ was obtained as a direct equilibrium measurement for binding of HPA. The $K_{d}{ }^{H P A}$ of wild-type $\mathrm{TK}_{\text {low }} K_{d(\text { low })}{ }^{H P A}$, was very similar to the previously reported inhibition constant of HPA, $K_{i}^{H P A 1,2}$, which suggested a possible relationship between $\mathrm{TK}_{\mathrm{low}}$ binding to HPA and substrate inhibition by HPA, despite the fact that the $K_{i}^{H P A}$ was obtained with no knowledge of the two-species model of transketolase.

HPA appeared to bind to the wild-type $\mathrm{TK}_{\text {high }}$ active-sites with slight positive cooperativity $(n=1.38 \pm 0.19)$, while HPA binding to $\mathrm{TK}_{\text {low }}$ was highly cooperative with a Hill coefficient of $3.10 \pm 0.16$. This high Hill coefficient suggests a potentially important role of $\mathrm{TK}_{\text {low }}$ inhibition by HPA in the regulation of TK activity.

Heat-induced activation and conversion of $\mathrm{TK}_{\mathrm{low}}$ to a $\mathrm{TK}_{\text {high }}$-like state. The previous study into heat-activation of TK used cofactor concentrations of $0.5 \mathrm{mM} \mathrm{Mg}{ }^{2+}$ and $50 \mu \mathrm{M} \mathrm{TPP}$, rather than $9 \mathrm{mM} \mathrm{Mg}^{2+14}$. At these concentrations, the [TPP] was semi-saturating for $\mathrm{TK}_{\text {high }}$ (approximately $60 \%$ saturated) but too low for $\mathrm{TK}_{\text {low }}$ saturation (approximately $20 \%$ saturated) 6 . In other words, the majority of $\mathrm{TK}_{\text {high }}$ was in the catalytically active holo-form while $\mathrm{TK}_{\mathrm{low}}$ was mostly in the catalytically inactive apo-form. Therefore, we hypothesised that heat exposure may convert $\mathrm{TK}_{\text {low }}$ to $\mathrm{TK}_{\mathrm{high}}$, hence increasing the concentration of holo-transketolase and overall activity of the sample.

We tested our hypothesis by taking fluorescence quenching measurements of $0.05 \mathrm{mg} / \mathrm{mL} \mathrm{TK}, 9 \mathrm{mM} \mathrm{Mg}^{2+}$ and a range of [TPP] before ${ }^{6}$ and after incubation at $42^{\circ} \mathrm{C}$ for 1 hour (Fig. 3b; Table 4). As hypothesised, the $\% B_{\max (\text { high }}$ increased from $33.7 \%$ to $51.7 \%$ after heat-activation. In addition, the affinity of both $\mathrm{TK}_{\text {high }}$ and $\mathrm{TK}_{\text {low }}$ increased significantly. Performing the same heat activation for a second time on the same sample increased the $\% B_{\max (h i g h)}$ only slightly to $53.5 \%$ (Fig. S5, Supplementary Information), indicating that no further change could be induced. Conversely, heat-activation had negligible impact on $\% \mathrm{TK}_{\text {modified }}(29.4 \pm 4.1 \%)$, determined from the mass spectra of heat-activated wild-type transketolase (Fig. 3c). Taken together, these results suggested heat-activation may occur through formation of a $\mathrm{TK}_{\text {high }}$-like conformational state but via a different mechanism to that of oxidation of $\mathrm{TK}_{\text {low }}$ to $\mathrm{TK}_{\mathrm{high}}$.

The activity of $0.05 \mathrm{mg} / \mathrm{mL}$ TK, $9 \mathrm{mM} \mathrm{Mg}^{2+}$ and $50 \mu \mathrm{M}$ TPP towards $50 \mathrm{mM} \mathrm{GA}$ and $50 \mathrm{mM}$ HPA pre- and post-incubation was determined to calculate the activity of heat-activated $\mathrm{TK}_{\mathrm{high}}$ relative to pre-incubated $\mathrm{TK}_{\text {high }}$ (Fig. 3d; Table 5). Overall, a $24.6 \%$ increase in transketolase activity was observed after heating, consistent with our hypothesis, though approximately only $50 \%$ of the activity enhancement expected from the $\% B_{\max (h i g h)}$ increase. This may indicate lower activity in the heat-induced $\mathrm{TK}_{\mathrm{high}}$-like state compared to the oxidised $\mathrm{TK}_{\mathrm{high}}$, or some partial unfolding and inactivation during heating. These and other possible mechanisms were not investigated further here.

The existence of a $\mathrm{TK}_{\text {high }}-\mathrm{TK}_{\text {low }}$ mixed dimer species that mediates HPA substrate inhibition. The equivalence between the $K_{d(\text { low })}{ }^{H P A}$ and the $K_{i}^{H P A}$ measured in previous activity assays suggested that binding of HPA to $\mathrm{TK}_{\mathrm{low}}$ gave rise to the observed overall inhibition of transketolase activity. However, the activity of $\mathrm{TK}_{\mathrm{low}}$ was already only $4.5 \%$ relative to that of $\mathrm{TK}_{\mathrm{high}}{ }^{6}$, implying that the $\mathrm{TK}_{\mathrm{low}}-\mathrm{TK}_{\mathrm{low}}$ dimer was effectively inactive already, and so binding of HPA to that dimer species could not have contributed significantly to the observed reaction inhibition. Therefore, the interaction between $\mathrm{HPA}$ and $\mathrm{TK}_{\mathrm{low}}$ must inhibit the $\mathrm{TK}_{\mathrm{high}}$ activity, which in turn suggested that inhibition occurred within a $\mathrm{TK}_{\mathrm{high}}-\mathrm{TK}_{\mathrm{low}}$ mixed dimer form. We therefore attempted to estimate the relative proportions of the three dimeric species, $\mathrm{TK}_{\mathrm{high}}-\mathrm{TK}_{\mathrm{high}}, \mathrm{TK}_{\mathrm{high}}-\mathrm{TK}_{\mathrm{low}}$, and $\mathrm{TK}_{\mathrm{low}}-\mathrm{TK}_{\mathrm{low}}$, from the heat-activation, HPA-binding, and enzyme activity data available.

Estimation of the \% dimer forms from heat-activation data. We first assumed that conversion of $\mathrm{TK}_{\mathrm{low}}$ to a $\mathrm{TK}_{\text {high }}$-like state via heat-activation was only possible for the proportion of $\mathrm{TK}_{\text {low }}$ present within a $\mathrm{TK}_{\text {high }}-\mathrm{TK}_{\text {low }}$ mixed dimer. We assumed all $\mathrm{TK}_{\mathrm{low}}$ subunits of the mixed dimer were completely converted to a $\mathrm{TK}_{\mathrm{high}}$-like state, and calculated the relative proportions of each dimeric species before heat-activation from the change in $\% B_{\max (h i g h)}$ upon heat-activation:

$$
\begin{aligned}
& \text { (A) } \left.\% \mathrm{TK}_{\text {high }}-\mathrm{TK}_{\text {low }}=\left(\% B_{\max (\text { high })}\right)^{\text {heated }}-\% B_{\max (\text { high })}^{\text {unheated }}\right) * 2=(51.7 \%-33.6 \%) * 2=36.2 \% \text {; } \\
& \text { (B) } \% \mathrm{TK}_{\text {high }}-\mathrm{TK}_{\mathrm{high}}=\% B_{\max (\text { high })}^{\text {unheated }}-(\mathrm{A}) / 2=33.6 \%-(36.2 / 2)=15.5 \% \text {; } \\
& \text { (C) } \% \mathrm{TK}_{\mathrm{low}}-\mathrm{TK}_{\mathrm{low}}=100 \%-(\mathrm{A})-(\mathrm{B})=100 \%-36.2 \%-15.5 \%=48.3 \% \text {. }
\end{aligned}
$$

Estimation of the \% dimer forms from HPA-inhibition data. Previous studies into the inhibition of transketolase determined the inhibition constants and the maximum inhibition of wild-type transketolase activity by HPA, $\% I_{\max }$, for wild-type $\left(K_{i}^{H P A}=43 \mathrm{mM} ; \% I_{\max }=48.1 \pm 5.1 \%\right)^{2}$ and also the D469T variant of transketolase $\left(K_{i}^{H P A}=40 \mathrm{mM} ; \% I_{\max }=46.8 \pm 11.5 \%\right)^{29}$.

Making the assumption that HPA-binding to the $\mathrm{TK}_{\mathrm{low}}$ subunit of the mixed dimer resulted in total inhibition of the $\mathrm{TK}_{\text {high }}$ subunit in that mixed dimer, and that $\mathrm{TK}_{\text {high }}$ accounted for $91 \%$ of total activity, we predicted the relative proportions of the three dimeric species:

$$
\begin{gathered}
\text { (A) } \% \mathrm{TK}_{\mathrm{high}}-\mathrm{TK}_{\mathrm{low}}=\left(\% B_{\max (\text { high })}^{\text {unheated }} *\left(\% I_{\max } / 91 \%\right) * 2=(33.6 \% *(48.1 \% / 91 \%) * 2=35.5 \%\right. \\
\text { (B) } \% \mathrm{TK}_{\mathrm{high}}-\mathrm{TK}_{\mathrm{high}}=\% B_{\max (\text { high })} \text { unheated }-(\mathrm{A}) / 2=15.8 \%
\end{gathered}
$$




\begin{tabular}{|l|l|l|}
\hline Dimer species & $\begin{array}{l}\text { \% Species (heat- } \\
\text { activation data) }\end{array}$ & $\begin{array}{l}\text { \% Species (inhibition } \\
\text { data) }\end{array}$ \\
\hline $\mathrm{TK}_{\text {high }}-\mathrm{TK}_{\text {high }}$ & $15.5 \%$ & $15.8 \%$ \\
\hline $\mathrm{TK}_{\text {high }}-\mathrm{TK}_{\text {low }}$ & $36.2 \%$ & $35.5 \%$ \\
\hline $\mathrm{TK}_{\text {low }}-\mathrm{TK}_{\text {low }}$ & $48.3 \%$ & $48.7 \%$ \\
\hline
\end{tabular}

Table 6. Summary of the predicted \% dimer of $\mathrm{TK}_{\text {high }}-\mathrm{TK}_{\mathrm{high}}, \mathrm{TK}_{\mathrm{high}}-\mathrm{TK}_{\mathrm{low}}$ and $\mathrm{TK}_{\text {low }}-\mathrm{TK}_{\mathrm{low}}$ calculated from TPP-binding data after heat activation and HPA-inhibition data. Note that the \% species from the HPA inhibition data accounts for $\mathrm{TK}_{\text {high }}$ having only $91 \%$ of total activity.

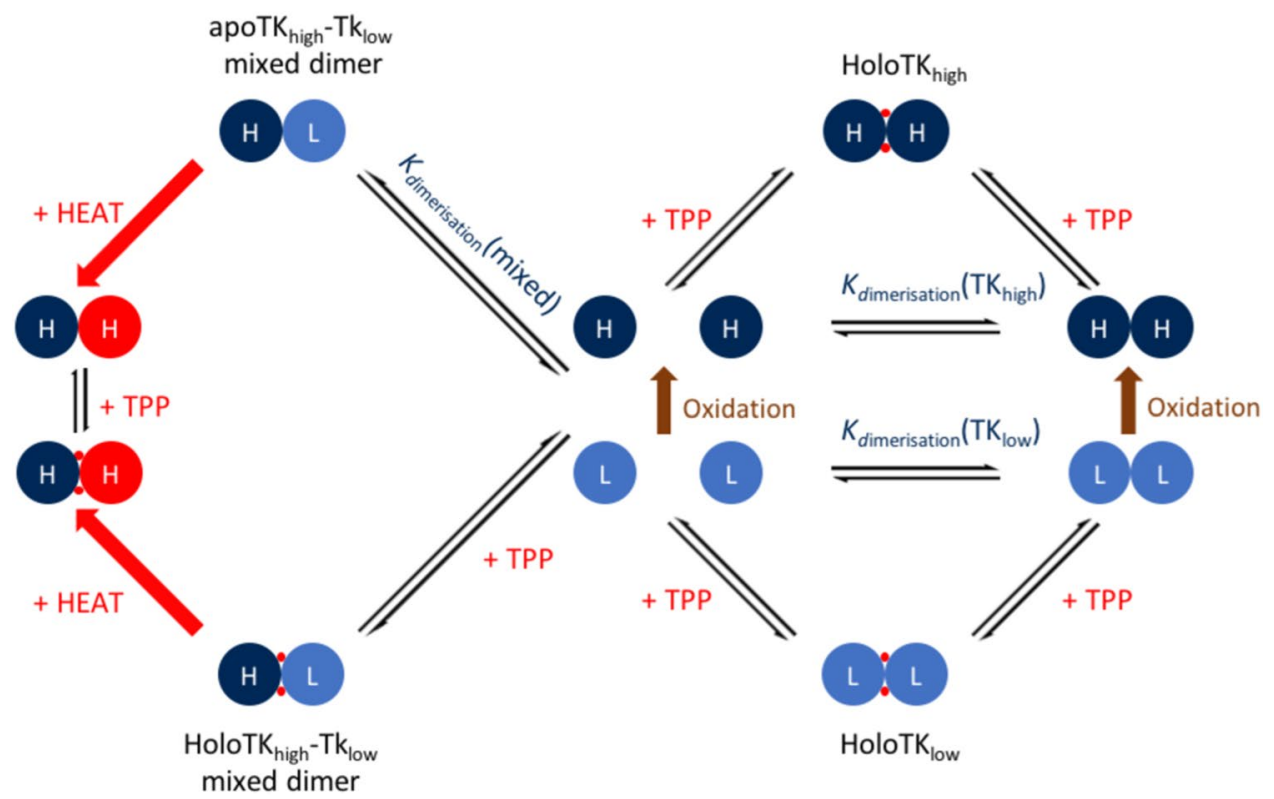

Figure 4. The updated Two-Species Model of transketolase activation, regulation and inhibition. The model is based on the combined TPP binding and AUC data before and after heat activation, as well as activity data. $\mathrm{L}$ (light blue) represents an inactive $\mathrm{TK}_{\mathrm{low}}$ monomer, $\mathrm{H}$ (navy blue) an active $\mathrm{TK}_{\text {high }}$ monomer, and $\mathrm{H}$ (red) a $\mathrm{TK}_{\text {high }}$-like monomer post-heat activation. The mechanism of conversion of $\mathrm{TK}_{\text {low }}$ to $\mathrm{TK}_{\text {high }}$ occurs through oxidation of Cys157 (brown).

$$
\text { (C) } \% \mathrm{TK}_{\mathrm{low}}-\mathrm{TK}_{\mathrm{low}}=100 \%-(\mathrm{A})-(\mathrm{B})=100 \%-35.5 \%-15.8 \%=48.7 \% \text {. }
$$

The similarity between the relative proportions of the three dimer species, calculated from the $\% I_{\max }$ obtained through activity data, and from TPP-binding data after heat-activation (Table 6), provides compelling evidence for our reasoning above that HPA binding to the $\mathrm{TK}_{\mathrm{low}}$ subunit within the mixed dimer resulted in inhibition of the associated $\mathrm{TK}_{\mathrm{high}}$ subunit in that mixed dimer. It also implies that heat-activation may happen via the removal of the donor substrate inhibition when the $\mathrm{TK}_{\mathrm{low}}$ subunit in the mixed dimer is converted into a $\mathrm{TK}_{\text {high }}$-like state. The fact that inhibition via the mixed dimer species persisted into a single-mutant TK variant, and with a different acceptor substrate, also suggested that this phenomenon is fundamental to the TK structure and mechanism when utilising HPA as the donor substrate.

The unified two-species model of transketolase activation, regulation and inhibition. The development of assays capable of detecting both $\mathrm{TK}_{\text {high }}$ and $\mathrm{TK}_{\mathrm{low}}$ has facilitated investigations into donor substrate inhibition, active site cooperativity and heat-activation, and finally led to the discovery of a novel mechanism of transketolase regulation. This can be summarised in the unified Two-Species Model of transketolase activation, regulation and inhibition (Fig. 4).

In summary:

- Transketolase exists as two distinct transketolase species; $\mathrm{TK}_{\mathrm{high}}$ and $\mathrm{TK}_{\mathrm{low}}$

- Inactive $\mathrm{TK}_{\text {low }}$ is the reduced, unmodified form of transketolase that is converted to $\mathrm{TK}_{\mathrm{high}}$ via oxidation of one or more methionine or cysteine residues, in response to cellular oxidative stress.

- $\quad \mathrm{TK}_{\mathrm{high}}$ is significantly more active and also has a 35-fold increased affinity for TPP at physiologically relevant $\left[\mathrm{Mg}^{2+}\right]$.

- Oxidation improves the cooperativity between active sites. Cys 157 oxidation is a strong candidate ${ }^{6}$, possibly by providing the final residue of a proton wire between active sites. 
- Redox regulation of transketolase potentially provides an important mechanism of control in diverting flux from glycolysis to the PPP during oxidative stress.

- Heat-activation of transketolase converts the $\mathrm{TK}_{\mathrm{low}}$ subunit of the mixed dimer to a $\mathrm{TK}_{\mathrm{high}}$-like state, which relieves the substrate inhibition of the associated $\mathrm{TK}_{\mathrm{high}}$ subunit.

- Heat-activation may offer cells a degree of heat shock protection by activating transketolase - a key enzyme in central metabolism - without the energetic or time-cost of protein biosynthesis.

\section{Discussion}

In this study, we attempted to resolve several unanswered questions remaining from our previous study into transketolase activation ${ }^{6}$; does the two-species phenomenon persist across variants and from cofactor to donor substrate; what is the cause of substrate inhibition; and what is the origin and physiological relevance of heat-activation?

Though wild-type, D469T and S385Y/D469T/R520Q all exist as a mix of $\mathrm{TK}_{\text {high }}$ and $\mathrm{TK}_{\text {low }}$ the susceptibility of $\mathrm{TK}_{\text {low }}$ to oxidation could potentially be enhanced through protein mutations in order to maximise [ $\mathrm{TK}_{\text {high }}$ ] and hence activity. Alternatively, controlled oxidation of purified transketolase samples may be possible, but this may require careful control to avoid over-oxidation of Cys157, or at other sites, that eventually would cause inactivation.

It is worth noting that this study and our previous study ${ }^{6}$ only investigated TPP binding in the presence/ absence of $\mathrm{Mg}^{2+}$ and not $\mathrm{Ca}^{2+}$. It is possible that $\mathrm{Ca}^{2+}$-reconstituted transketolase is activated differently via different oxidation-activation pathways. However, all mass spectra in these studies were derived from transketolases in their apo-form and the formation of modified, oxidised species were hence cation-independent. It is both possible that $\mathrm{Ca}^{2+}$-reconstituted transketolase is activated in a similar way to $\mathrm{Mg}^{2+}$-constituted transketolase, or active site coordination with $\mathrm{Ca}^{2+}$ bypasses the requirement of oxidation for activation. Further studies into TPP- and HPA binding to $\mathrm{TK}_{\mathrm{high}}$ and $\mathrm{TK}_{\mathrm{low}}$ in the presence of $\mathrm{Ca}^{2+}$ would be able to determine which hypothesis is correct, but are beyond the scope of this study.

It is interesting that HPA binding to $\mathrm{TK}_{\text {low }}$ had such a high degree of positive cooperativity, and may indeed result from multiple HPA molecules binding to a single active-site to inhibit activity in that active site. Furthermore, it may also inhibit active-site synchronisation by inhibiting the shuttling of protons along the proton wire. The existence of the $\mathrm{TK}_{\mathrm{high}}-\mathrm{TK}_{\mathrm{low}}$ mixed dimer potentially complicates further the analysis of Hill coefficients for $\mathrm{TK}_{\text {high }}$ and $\mathrm{TK}_{\text {low }}$. However, the true Hill-coefficient of $\mathrm{TK}_{\text {high }}$ is likely to be higher than the reported apparent value, whereas the true Hill-coefficient of $\mathrm{TK}_{\mathrm{low}}$ is presumably lower. The most abundant dimer, $\mathrm{TK}_{\mathrm{low}}-\mathrm{TK}_{\mathrm{low}}$, may also have previously masked the recently-elucidated asymmetric structure of the TK apo-dimer ${ }^{13}$, given the reduced cooperativity of TPP-binding between active sites in this dimer.

The heat-activation of TK appeared to increase activity through a slightly different mechanism to that of oxidation of $\mathrm{TK}_{\text {low }}$ to $\mathrm{TK}_{\mathrm{high}}$. Conformational rearrangements might result from heat-activation and give rise to a $\mathrm{TK}_{\text {high }}$-like conformational state that apparently relieves HPA inhibition of the $\mathrm{TK}_{\mathrm{high}}$ subunit of the mixed dimer. The heat-activation of transketolase may even be part of the cellular response to thermal stress, resulting in the upregulation of the PPP and hence NADPH production, while also increasing flux through critical biosynthetic pathways such as nucleotide biosynthesis. The heat-sensitivity of transketolase therefore has a potential role in facilitating rapid global changes in metabolism without the additional energetic burden of protein synthesis.

Finally, the discovery of a redox-sensitive regulatory system that activates transketolase, potentially during oxidative stress, could have important implications for future advances in cancer treatments. Cancer cells are often exposed to higher levels of oxidative stress than normal cells, and elevated transketolase activity has been reported in a number of cancer types in order to reduce ultimately catastrophic damage from high oxidative stress $^{30}$. The redox-sensitivity of transketolase not only makes transketolase itself an antioxidant, but also an important mediator of the biosynthesis of a second anti-oxidant, NADPH. Reversal or prevention of transketolase oxidation at residue Cys 157 by drug delivery or gene therapy may offer an effective way to prevent antioxidant production and hence proliferation in cancer cells.

\section{Methods}

Materials. TPP, $\mathrm{MgCl}_{2}$, glycolaldehyde (GA) and Erythrulose [Ery] were purchased from Sigma-Aldrich; Tris- $\mathrm{HCl}$ was purchased from VWR International and Guanidine-HCL was purchased from Life Technologies Ltd. HPA was synthesised from bromopyruvic acid and $\mathrm{LiOH}$, as described previously ${ }^{31}$.

Enzyme preparation. Wild-type transketolase with an $\mathrm{N}$-terminal His6 tag was expressed in E. coli XL10-gold cells (Agilent Technologies Ltd) from the plasmid pQR791. The resulting cell pellet was lysed and purified as described previously ${ }^{32}$. Purified transketolase was ultrafiltrated four times using an Amicon Ultra-4 10k MWCO (Millipore, US) centrifugal filter to remove excess imidazole and cofactors and subsequently dialysed overnight at $4{ }^{\circ} \mathrm{C}$ in $50 \mathrm{mM}$ Tris- $\mathrm{HCl}, \mathrm{pH} 7.0$ to obtain apo-TK. Protein concentration was determined by absorbance at $280 \mathrm{~nm}$ in $6 \mathrm{M}$ Guanidine- $\mathrm{HCl}$ and $20 \mathrm{mM}$ Sodium Phosphate, $\mathrm{pH}$ 6.5. Absorbance was measured using a Nanodrop spectrophotometer (Thermo Fisher Scientific, Wilmington, DE), assuming a monomeric molecular weight of $73035.5 \mathrm{~g} \mathrm{~mol}^{-1}$ and an extinction coefficient $(\varepsilon)$ of $92630 \mathrm{~L} \mathrm{~mol}^{-1} \mathrm{~cm}^{-1}$.

For TPP-binding assays, series of $2 \mathrm{x}$ concentrated cofactor solutions were prepared and purified TK was added to a final concentration of $0.05 \mathrm{mg} / \mathrm{mL}$. The samples were incubated at $22^{\circ} \mathrm{C}$ for 45 minutes to allow TK-TPP binding to reach equilibrium. For HPA-binding assays, $2 \mathrm{x}$ concentrated, purified holoTK was prepared at $0.1 \mathrm{mg} /$ $\mathrm{mL} \mathrm{TK}, 18 \mathrm{mM} \mathrm{Mg}^{2+}$ and $0.6 \mathrm{mM} \mathrm{TPP}$ and incubated at $22^{\circ} \mathrm{C}$ for 45 minutes to allow TK-TPP binding to reach equilibrium. The purified holoTK was added to a series of $2 \mathrm{x}$ concentrated HPA solutions and incubated at $22^{\circ} \mathrm{C}$ for 10 minutes to allow holoTK-HPA binding to reach equilibrium. For heat-activation studies, TK samples were 
incubated at $42^{\circ} \mathrm{C}$ for 1 hour and subsequently re-equilibrated at $4{ }^{\circ} \mathrm{C}$ for 30 minutes and at $22^{\circ} \mathrm{C}$ for 30 minutes prior to assays.

Fluorescence assay to detect TPP binding. TPP-binding was measured using a Fluoromax-4 (Horiba, UK) spectrofluorometer $\left(\lambda_{\mathrm{ex}}=240 \mathrm{~nm} ; \lambda_{\mathrm{em}}=330 \mathrm{~nm}\right.$; integration time $=0.1 \mathrm{~s}$; slit width $\left.=8 \mathrm{~nm}\right)$, as described previously ${ }^{6}$.

Transketolase activity assay. Purified, dialysed apo-transketolase $(0.2 \mathrm{mg} / \mathrm{mL})$ was incubated with $2.4 \mathrm{mM}$ TPP and $9 \mathrm{mM} \mathrm{Mg}^{2+}$ for 45 minutes at $22^{\circ} \mathrm{C} .50 \mu \mathrm{L}$ was added to $100 \mu \mathrm{L} 150 \mathrm{mM} \mathrm{GA}, 150 \mathrm{mM} \mathrm{HPA}$, giving final substrate concentrations of $50 \mathrm{mM}$. The reaction was performed in triplicate at $22^{\circ} \mathrm{C}$ in a 96 well plate with shaking at $300 \mathrm{rpm}$ using a Thermomixer Comfort shaker. $10 \mu \mathrm{L}$ of the reaction was quenched with $190 \mu \mathrm{L}$ $0.1 \%$ trifluoroacetic acid (TFA) after 3, 5, 10, 15, 20,30, and 40 minutes. Samples were subsequently analysed by a Dionex HPLC system (Camberley, UK) with a Bio-Rad Aminex HPX-87H reverse phase column $(300 \times 7.8$ $\mathrm{mm}^{2}$ ) (Bio-Rad Labs., Richmond, CA, USA), via Chromeleon client 6.60 software, to separate and analyse the change in the concentration of substrate (GA) and product (Ery) over the course of the reaction using the method described previously ${ }^{29}$.

Fluorescence assay to detect HPA binding. The same methodology was used as the TPP-binding fluorescence assay, except cofactor concentrations were kept constant at $9 \mathrm{mM} \mathrm{Mg}^{2+}$ and $0.3 \mathrm{~mm} \mathrm{TPP}$, and an IFE CF was generated between $0-80 \mathrm{mM}$ HPA.

Mass spectrometry (LC-ESI-MS). LC-MS was performed using an Agilent 1100/1200 LC system connected to a 6510 A QTOF mass spectrometer (Agilent, UK), as described previously ${ }^{6}$. Triplicate fermentations were performed for each sample.

\section{Data availability}

The data that support the findings of this study are available from the corresponding author upon reasonable request.

Received: 13 November 2019; Accepted: 22 February 2020;

Published online: 05 March 2020

\section{References}

1. Gyamerah, M. \& Willetts, A. J. Kinetics of overexpressed transketolase from Escherichia coli JM 107/pQR 700. Enzyme Microb. Technol. 20, 127-134 (1997).

2. Chen, B. H., Hibbert, E. G., Dalby, P. A. \& Woodley, J. M. A new approach to bioconversion reaction kinetic parameter identification. 54, 2155-2163 (2008).

3. Lindqvist, Y., Schneider, G. \& Vihko, P. Three-dimensional structure of rat acid phosphatase in complex with L(+)- tartrate. J. Biol. Chem. 268, 20744-20746 (1993).

4. Littlechild, J. et al. Crystallization and preliminary X-ray crystallographic data with Escherichia coli transketolase. Acta Crystallogr. Sect. D Biol. Crystallogr. 51, 1074-1076 (1995).

5. Solovjeva, O. N., Selivanov, V. A., Orlov, V. N. \& Kochetov, G. A. Stages of the formation of nonequivalence of active centers of transketolase from baker's yeast. Mol. Catal. (2019).

6. Wilkinson, H. C. \& Dalby, P. A. Novel insights into transketolase activation by cofactor binding identifies two native species subpopulations. Sci. Rep. $\mathbf{9}$ (2019).

7. Sevostyanova, I. A. et al. Cooperative binding of substrates to transketolase from Saccharomyces cerevisiae. Biochem. Biokhimiĩa 74, 789-92 (2009).

8. Seifert, F. et al. Direct kinetic evidence for half-of-the-sites reactivity in the E1 component of the human pyruvate dehydrogenase multienzyme complex through alternating sites cofactor activation. Biochemistry 45, 12775-12785 (2006).

9. Jordan, F., Nemeria, N. S. \& Sergienko, E. Multiple modes of active center communication in thiamin diphosphate-dependent enzymes. Acc. Chem. Res. 38, 755-763 (2005).

10. Nemeria, N. S. et al. Communication between thiamin cofactors in the Escherichia coli pyruvate dehydrogenase complex E1 component active centers: evidence for a "direct pathway" between the 4'-aminopyrimidine N1' atoms. J. Biol. Chem. 285, 11197-209 (2010).

11. Schröder-Tittmann, K. et al. Alternating sites reactivity is a common feature of thiamin diphosphate-dependent enzymes as evidenced by isothermal titration calorimetry studies of substrate binding (2013).

12. Frank, R. A. W., Titman, C. M., Pratap, J. V., Luisi, B. F. \& Perham, R. N. A molecular switch and proton wire synchronize the active sites in thiamine enzymes. Science (80-). 306, 872-876 (2004).

13. Dai, S. et al. Low-barrier hydrogen bonds in enzyme cooperativity. Nature 573, 609-613 (2019).

14. Jahromi, R. R. F., Morris, P., Martinez-Torres, R. J. \& Dalby, P. A. Structural stability of E. coli transketolase to temperature and $\mathrm{pH}$ denaturation. J. Biotechnol. 155, 209-216 (2011).

15. Morris, P., Rios-Solis, L., García-Arrazola, R., Lye, G. J. \& Dalby, P. A. Impact of cofactor-binding loop mutations on thermotolerance and activity of E. coli transketolase. Enzyme Microb. Technol. 89, 85-91 (2016).

16. Kuehne, A. et al. Acute activation of oxidative pentose phosphate pathway as first-line response to oxidative stress in human skin cells. Mol. Cell 59, 359-371 (2015).

17. Pai, E. F. \& Shultz, G. E. The catalytic mechanism of glutathione reductase as derived from X-ray diffraction analyses. J. Biol. Chem. 258, 1752-1757 (1983).

18. Rescigno, M. \& Perham, R. N. Structure of the NADPH-binding motif of glutathione reductase: efficiency determined by evolution. Biochemistry 33 (1994).

19. Stincone, A. et al. The return of metabolism: biochemistry and physiology of the pentose phosphate pathway. Biol. Rev. 90, 927-963 (2015).

20. Anastasiou, D. et al. Inhibition of pyruvate kinase M2 by reactive oxygen species contributes to cellular antioxidant responses. Science (80-). 334, 1278-1283 (2011).

21. Colussi, C. et al. $\mathrm{H}_{2} \mathrm{O}_{2}$-induced block of glycolysis as an active ADP-ribosylation reaction protecting cells from apoptosis. FASEB J. 14, 2266-2276 (2000).

22. Ralser, M. et al. Dynamic rerouting of the carbohydrate flux is key to counteracting oxidative stress. J. Biol. 6 (2007). 
23. Ralser, M. et al. Metabolic reconfiguration precedes transcriptional regulation in the antioxidant response. Nat. Biotechnol. 27, 604-605 (2009).

24. Zhang, Y., Wang, M. \& Lin, H. A regulatory cysteine residue mediates reversible inactivation of $\mathrm{NAD}^{+}$-dependent aldehyde dehydrogenases to promote oxidative stress response. ACS Chem. Biol. 15, 28-32 (2020).

25. Comín-Anduix, B. et al. The effect of thiamine supplementation on tumour proliferation: A metabolic control analysis study. Eur. J. Biochem. 268, 4177-4182 (2001).

26. Payongsri, P., Steadman, D., Hailes, H. C. \& Dalby, P. A. Second generation engineering of transketolase for polar aromatic aldehyde substrates. Enzyme Microb. Technol. 71, 45-52 (2015).

27. Yi, D. et al. A pH-based high-throughput assay for transketolase: fingerprinting of substrate tolerance and quantitative kinetics. ChemBioChem 13, 2290-2300 (2012).

28. Hibbert, E. G. et al. Directed evolution of transketolase activity on non-phosphorylated substrates. J. Biotechnol. 131, 425-432 (2007).

29. Chen, B. H., Micheletti, M., Baganz, F., Woodley, J. M. \& Lye, G. J. An efficient approach to bioconversion kinetic model generation based on automated microscale experimentation integrated with model driven experimental design. Chem. Eng. Sci. 64, 403-409 (2009).

30. Xu, I. M.-J. et al. Transketolase counteracts oxidative stress to drive cancer development. Proc. Natl. Acad. Sci. 113, E725-E734 (2016).

31. Morris, K. G. et al. Transketolase from Escherichia coli: A practical procedure for using the biocatalyst for asymmetric carboncarbon bond synthesis. Tetrahedron Asymmetry 7, 2185-2188 (1996).

32. Martinez-Torres, R. J., Aucamp, J. P., George, R. \& Dalby, P. A. Structural stability of E. coli transketolase to urea denaturation. Enzyme Microb. Technol. 41, 653-662 (2007).

\section{Acknowledgements}

We thank Dr. Kersti Karu for mass spectrometric measurements in the UCL Department of Chemistry Mass Spectrometry Facility, and Dr. John Ward for donation of the transketolase plasmid pQR791. We thank the Engineering and Physical Sciences Research Council (EPSRC), and their Centre for Doctoral Training in Emergent Macromolecular Therapies, for providing our grant (EP/L015218/1).

\section{Author contributions}

H.C.W. carried out all experimental procedures, unless stated otherwise. P.A.D. and H.C.W. wrote the main manuscript text and H.C.W. prepared all figures. All authors reviewed the manuscript.

\section{Competing interests}

The authors declare no competing interests.

\section{Additional information}

Supplementary information is available for this paper at https://doi.org/10.1038/s41598-020-61175-z.

Correspondence and requests for materials should be addressed to P.A.D.

Reprints and permissions information is available at www.nature.com/reprints.

Publisher's note Springer Nature remains neutral with regard to jurisdictional claims in published maps and institutional affiliations.

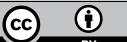

Open Access This article is licensed under a Creative Commons Attribution 4.0 International License, which permits use, sharing, adaptation, distribution and reproduction in any medium or format, as long as you give appropriate credit to the original author(s) and the source, provide a link to the Creative Commons license, and indicate if changes were made. The images or other third party material in this article are included in the article's Creative Commons license, unless indicated otherwise in a credit line to the material. If material is not included in the article's Creative Commons license and your intended use is not permitted by statutory regulation or exceeds the permitted use, you will need to obtain permission directly from the copyright holder. To view a copy of this license, visit http://creativecommons.org/licenses/by/4.0/.

(C) The Author(s) 2020 